\title{
A New Decomposition Formula in the Theory of Elasticity ${ }^{1}$
}

\author{
J. H. Bramble ${ }^{2}$ and L. E. Payne ${ }^{2}$
}

(February 17, 1961)

\begin{abstract}
In this paper a new representation formula for the spherical components of displacement in the theory of elasticity is obtained. This formula may be used to reduce mixed boundary value problems for the elastic sphere to standard problems in potential theory.
\end{abstract}

\section{Introduction}

In treating boundary value problems in the theory of elasticity it is frequently convenient to represent the components of the displacement vector in terms of harmonic functions, and thus to reduce the elasticity problem to one in potential theory. Several such decompositions in terms of harmonic functions are known, [7, p. 342-343], ${ }^{3}$ [1, p. 231], [4], the most widely used, perhaps, being the Papkovitch-Neuber decomposition (see for instance [7, p. 331]).

Half-space problems have been quite thoroughly treated from this point of view, as in fact have the first and second boundary value problems for the sphere. However, in treating mixed boundary value problems for the sphere, the standard decompositions are not suitable. Such mixed problems could be simplified considerably if it were possible to obtain convenient representations for the spherical components of displacement in terms of harmonic functions. We obtain such a representation in this paper and demonstrate how it yields in a simple manner solutions to standard mixed boundary value problems for the sphere.

In this paper whenever an index is repeated in a single term, summation from 1 to 3 will be implied. Use of the symbol $f_{, i}$ to denote $\partial f / \partial x_{i}$, where $x_{i}$ is one of the rectangular coordinates $\left(x_{1}, x_{2}, x_{3}\right)$, will be made throughout.

Let $D$ be a three dimensional region which contains an open subset $Q$ of a spherical surface of radius a with center at the origin of a spherical coordinate system $(r, \theta, \varphi)$. The region $D$ is assumed to be a portion of an isotropic homogeneous elastic medium.

We suppose that $D$ does not contain the origin. Furthermore, we assume that every ray from the origin which intersects $D$, intersects it in a single line segment.

In $D$ the component of the displacement vector satisfies the equilibrium equations

$$
u_{i, j j}+\alpha u_{j, j i}=0
$$

where $\alpha$ involves the elastic constants $\left(\alpha=(1-2 \sigma)^{-1}\right.$ where $\sigma$ is Poisson's ratio). As far as our representation formulas are concerned we need put no restrictions on the constant $\alpha$ other than that it be different from -1 . However, all physically interesting values of $\alpha$ lie in the interval $\frac{1}{3}<\alpha<\infty$.

\footnotetext{
1 This research was supported in part by the U.S. Air Force through the Air Force Office of Scientific Research of the Air Research and Development Command under Contract No. A F 49(638)-228.

2 University of Maryland, College Park, Md.

3 Figures in brackets indicate the literature references at the end of this paper.
} 
In this paper we obtain the following representation for the spherical components of displacement in $D$ :

$$
\begin{gathered}
x_{j} u_{j}=r^{2}\left\{2 G+(2-\alpha) r \partial G / \partial r-\alpha r \frac{\partial}{\partial r}\left(r \frac{\partial G}{\partial r}\right)\right\}+r \frac{\partial \psi}{\partial r} \\
\epsilon_{i j k} x_{j} u_{k}=r^{2}\left\{-(2+3 \alpha) \epsilon_{i j k} x_{j} G, k-\alpha r \frac{\partial}{\partial r} \epsilon_{i j k} x_{j} G_{, k}-\left(H+r \frac{\partial H}{\partial r}\right)_{, i}\right\} \\
+\left\{x_{i}\left[r \frac{\partial H}{\partial r}+2 r \frac{\partial}{\partial r}\left(r \frac{\partial H}{\partial r}\right)\right]-r^{2}\left[r \frac{\partial H}{\partial r}\right]_{, i}\right\}+\epsilon_{i j k} x_{j} \psi_{, k}
\end{gathered}
$$

where $\psi, G$, and $H$ are harmonic functions in $D$. Consequently all terms in braces in (1.2) and (1.3) are also harmonic. In (1.3) $\epsilon_{i j k}$ is the permutation symbol

$$
\epsilon_{i j k}=\left\{\begin{aligned}
0 & \text { if any two indices are equal } \\
1 & \text { if }{ }_{i j k} \text { is an even permutation of } 123 \\
-1 & \text { if }{ }_{i j k} \text { is an odd permutation of } 123 .
\end{aligned}\right.
$$

\section{Representation Formulas}

We first rewrite (1.1) as

$$
\epsilon_{i j k} \frac{\partial}{\partial x_{j}} \epsilon_{k l m} u_{l, m}+(\alpha+1) u_{j, j i}=0 .
$$

For $\alpha \neq-1$ we see from (1.1) that $u_{j}, j$ is harmonic. It has been shown that the representation

$$
u_{j, j}=\left(1+r \frac{\partial}{\partial r}\right)\left(6 G+4 r \frac{\partial G}{\partial r}\right)
$$

where $G$ is harmonic, is valid in $D$ (see for instance Bramble [2]). Equation (2.1) then may be integrated to give

$$
\boldsymbol{\epsilon}_{i j k} u_{j, k}=(\alpha+1) \boldsymbol{\epsilon}_{i j k} x_{j}\left(6 G+4 r \frac{\partial G}{\partial r}\right)_{, k}+\chi,_{i}
$$

where $\chi$ is a harmonic function. We express $\chi$ in $D$ as

$$
\chi=\Phi+r \frac{\partial \Phi}{\partial r} .
$$

Thus $u_{j}$ is the component of a vector whose divergence is represented by (2.2) and whose curl is expressible by (2.3). The component $u_{j}$ may clearly be expressed as the sum of a particular solution of (2.2) and (2.3) and the gradient of a harmonic function, i.e.,

$$
u_{j}=2 x_{j}\left[G+(\alpha+2) r \frac{\partial G}{\partial r}\right]-r^{2}\left[2(1+2 \alpha) G_{, j}+\alpha r \frac{\partial}{\partial r} G_{. j}\right]+\epsilon_{j i k} x_{i} \Phi_{, k}+\psi_{, j}
$$

where $\psi$ is harmonic in $D$.

Using (2.5) we now form the expressions for $x_{j} u_{j}$ and $\epsilon_{i j k} x_{j} u_{k}$ which are respectively the scalar product and the vector product of the radius vector and the displacement vector.

$$
x_{j} u_{\boldsymbol{j}}=r^{2}\left\{2 G+(2-\alpha) r \frac{\partial G}{\partial r}-\alpha r \frac{\partial}{\partial r}\left(r \frac{\partial G}{\partial r}\right)\right\}+r \frac{\partial \psi}{\partial r}
$$

and

$$
\boldsymbol{\epsilon}_{i j k} x_{j} u_{k}=r^{2}\left\{-(2+3 x) \boldsymbol{\epsilon}_{i j k} x_{j} G_{, k}-\alpha r \frac{\partial}{\partial r} \epsilon_{i j k} x_{j} G_{, k}-\Phi_{i}\right\}+x_{i} r \frac{\partial \Phi}{\partial r}+\epsilon_{i j k} x_{j} \psi_{, k} .
$$


Equation (2.6) is precisely the desired expression (1.2). In order to obtain (1.3) we substitute for $\Phi$ in $D$ (see Bramble [2]),

$$
\Phi=H+2 r \frac{\partial H}{\partial r}
$$

where $H$ is harmonic. We then write

$$
x_{i} r \frac{\partial \Phi}{\partial r}=\left[x_{i}\left\{r \frac{\partial H}{\partial r}+2 r \frac{\partial}{\partial r}\left(r \frac{\partial H}{\partial r}\right)\right\}-r^{2}\left(r \frac{\partial H}{\partial r}\right)_{, i}\right]+r^{2}\left(r \frac{\partial H}{\partial r}\right)_{, i} .
$$

As noted previously, the term in square brackets is harmonic. Equations (2.7), (2.8) and (2.9) yield immediately the desired representation (1.3).

In obtaining our decompositions we have frequently made use of the fact that in the region in question any harmonic function could be represented as $\left(1+a_{1} r \frac{\partial}{\partial r}\right)\left(1+a_{2} r \frac{\partial}{\partial r}\right) g$ where $g$ is harmonic. We have used only representations in which $a_{1}$ and $a_{2}$ were non-negative. Bramble [2] has shown that such a representation is valid in $D$ regardless of the sign of $a_{1}$ and $a_{2}$. However, for $a_{1}$ and $a_{2}$ non-negative it is easily verified that such a representation is also valid in any region whose boundery is star-shaped with respect to the origin. Consequently (1.2) and (1.3) are valid in any such star shaped region. In the next section we use the representations (1.2) and (1.3) to solve certain boundary value problems for the elastic sphere.

If the elastic region is exterior to a surface $C$ which is star-shaped with respect to the origin and if the components of the displacement vector are $\mathrm{O}\left(r^{-2}\right)$ as $r \rightarrow \infty$, it can again be shown that the decompositions (1.2) and (1.3) are valid therein.

\section{Mixed Boundary Value Problems for the Sphere}

Let $u_{i}$ satisfy (1.1) on the interior of an elastic sphere. On the surface $r=a$ let

$$
\begin{array}{r}
\mu^{-1} a_{1} \tau_{i j} x_{i} x_{j}+a_{2} x_{j} u_{j}=f(\theta, \varphi) \\
\mu^{-1} b_{1} \epsilon_{i j k} \tau_{k l} x_{j} x_{l}+b_{2} \epsilon_{i j k} x_{j} u_{k}=g_{i}(\theta, \varphi)
\end{array}
$$

where $\tau_{i j}$ is the stress tensor, $\mu$ is the shear modulus, and where $f$ and $g_{i}$ are prescribed boundary data. Equations (3.1) may be written equivalently as

$$
\begin{gathered}
a_{1}(\alpha+1) r \frac{\partial}{\partial r}\left(x_{j} u_{j}\right)+\left[a_{1}(\alpha-3)+a_{2}\right] x_{j} u_{j}+a_{1}(\alpha-1) \epsilon_{i j k} x_{i} \frac{\partial}{\partial x_{j}} \epsilon_{k l m} x_{l} u_{m}=f(\theta, \varphi) \\
b_{1} r \frac{\partial}{\partial r} \epsilon_{i j k} x_{j} u_{k}+\left(b_{2}-2 b_{1}\right) \epsilon_{i j k} x_{j} u_{k}+b_{1} \epsilon_{i j k} x_{j}\left(x_{l} u_{l}\right)_{, k}=g_{i}(\theta, \varphi)
\end{gathered}
$$

on $r=a$. Using (1.2) and (1.3) we can actually obtain the following representation for the left hand side of (3.1) in terms of harmonic functions

$$
\begin{aligned}
\mu^{-1} a_{1} \tau_{i j} x_{i} x_{j}+a_{2} x_{j} u_{j}=r^{2}\{ & -2 a_{1} \alpha\left(r \frac{\partial}{\partial r}\right)^{3} G-a_{2} \alpha\left(r \frac{\partial}{\partial r}\right)^{2} G+\left[2 a_{1}(4 \alpha-1)+a_{2}(2-\alpha)\right] r \frac{\partial G}{\partial r} \\
& \left.+2\left[(3 \alpha-1) a_{1}+a_{2}\right] G\right\}+2 a_{1} r \frac{\partial}{\partial r}\left(r \frac{\partial \psi}{\partial r}\right)-\left(2 a_{1}-a_{2}\right) r \frac{\partial \psi}{\partial r}
\end{aligned}
$$


From (3.3) we obtain expressions of the form

$$
\begin{aligned}
& A_{1}\left(r \frac{\partial}{\partial r}\right)^{3} G+A_{2}\left(r \frac{\partial}{\partial r}\right)^{2} G+A_{3} r \frac{\partial G}{\partial r}+A_{4} G+A_{5} r \frac{\partial}{\partial r}\left(r \frac{\partial \psi}{\partial r}\right)+A_{6} r \frac{\partial \psi}{\partial r}=P(f) \\
& \epsilon_{i j k} x_{j} \frac{\partial}{\partial x_{k}}\left\{B_{1}\left(r \frac{\partial}{\partial r}\right)^{2} G+B_{2} r \frac{\partial G}{\partial r}+B_{3} G+B_{4} r \frac{\partial \psi}{\partial r}+B_{5} \psi\right\} \\
& +\left\{x_{i}\left(2 r \frac{\partial}{\partial r}+1\right)\left(r \frac{\partial}{\partial r}\right)\left(B_{6} r \frac{\partial H}{\partial r}+B_{7} H\right)-r^{2}\left[r \frac{\partial}{\partial r}\left(B_{6} r \frac{\partial H}{\partial r}+B_{7} H\right)\right]_{i}\right\} \\
& -a^{2}\left[\left(1+r \frac{\partial}{\partial r}\right)\left(B_{6} r \frac{\partial H}{\partial r}+B_{7} H\right)\right]_{, i}=P\left(g_{i}\right)
\end{aligned}
$$

valid throughout $D$. The constants $A_{i}$ and $B_{i}$ are determined immediately from (3.3) and $P(f)$ and $P\left(g_{i}\right)$ denote Poisson integrals.

Forming $x_{i} P\left(g_{i}\right)$ we obtain the expression

$$
B_{6}\left\{\left(r \frac{\partial}{\partial r}\right)^{3} H+\left(r \frac{\partial}{\partial r}\right)^{2} H\right\}+B_{7}\left\{\left(r \frac{\partial}{\partial r}\right)^{2} H+r \frac{\partial H}{\partial r}\right\}=\frac{x_{i} P\left(g_{i}\right)}{r^{2}-a^{2}}
$$

Under suitable conditions on $B_{6}$ and $B_{7}$ this expression may be integrated to give an expression for $H$. With $H$ known throughout $\mathrm{D}$, equations (3.4) give two identities in the two unknowns $G$ and $\psi$. The function $\psi$ can be eliminated by taking the proper combination of $r$ derivatives of the first of equations (3.4) and $r$ derivatives of the expression formed by operating on the second of equations (3.4) with $\epsilon_{l m i} x_{l} \frac{\partial}{\partial x_{m}}$. This results in an equation involving a linear combination of $r$ derivatives of $G$. If this equation can be solved for $G$ then the insertion of $G$ into the first of equations (3.4) yields the expression for $\psi$ which completely determines the displacement vector in $D$. It is a simple matter to obtain a formal solution for $G$, for we have an equation of the form

$$
\sum_{i=0}^{N} a_{i}\left(r \frac{\partial}{\partial r}\right)^{i} G=F
$$

We expand the known harmonic function $F$ in terms of spherical harmonics, i.e.,

$$
\sum_{i=0}^{N} a_{i}\left(r \frac{\partial}{\partial r}\right)^{i} G=\sum_{n=0}^{\infty} \sum_{m=0}^{n} r^{n} P_{n}^{m}(\cos \theta)\left[A_{n m} \cos m \phi+B_{n m} \sin m \phi\right] .
$$

The solution to this equation is

$$
G=\sum_{n=0}^{\infty} \sum_{m=0}^{n}\left[\sum_{i=0}^{N} a_{i} n^{i}\right]^{-1} r^{n} P_{n}^{m}(\cos \theta)\left[A_{n m} \cos m \phi+B_{n m} \sin m \phi\right]
$$

provided

$$
\sum_{i=0}^{N} a_{i} n^{i} \neq 0
$$

for $n=0,1,2, \ldots$, or any positive integer. For a given $\alpha,(3.9)$ imposes certain restrictions on the constants $a_{1}, a_{2}, b_{1}$, and $b_{2}$.

As a particular example consider the case in which $a_{2}$ and $b_{1}$ in (3.1) are zero. The constants $a_{1}$ and $b_{2}$ can without loss be taken as unity. Thus equation (3.4) becomes

$$
a^{2}\left[-2 \alpha\left(r \frac{\partial}{\partial r}\right)^{3} G+2(4 \alpha+1) r \frac{\partial G}{\partial r}+2(3 \alpha-1) G\right]+2 r \frac{\partial}{\partial r}\left(r \frac{\partial}{\partial r}-1\right) \psi=P(f)
$$


and

$$
\begin{aligned}
a^{2} \epsilon_{i j k} x_{j} \frac{\partial}{\partial x_{k}}\left[-\alpha r \frac{\partial G}{\partial r}-(2+3 \alpha) G\right]-\epsilon_{i j k} x_{j} \psi_{, k}-a^{2}\left(H+r \frac{\partial H}{\partial r}\right)_{, i} & \\
+ & \left\{x_{i}\left[r \frac{\partial H}{\partial r}+2\left(r \frac{\partial}{\partial r}\right)^{2} H\right]-r^{2}\left(r \frac{\partial H}{\partial r}\right)_{, i}\right\}=P\left(g_{i}\right)
\end{aligned}
$$

where $P\left(g_{i}\right)$ and $P(f)$ denote Poisson's integrals. If we take the scalar product of the radius vector and (3.11) we obtain the differential equation for $H$

$$
r \frac{\partial}{\partial r}\left[H+r \frac{\partial H}{\partial r}\right]=\frac{x_{i} P\left(g_{i}\right)}{r^{2}-a^{2}}
$$

which yields the result

$$
H=\int_{0}^{r} \rho^{-1} \frac{(r-\rho) x_{i} P\left(g_{\imath}\right)}{\left(\rho^{2}-a^{2}\right)} d \rho .
$$

It is possible to add an arbitrary constant to the right hand side of (3.13) but since only derivatives of $H$ occur in (1.3) we may take the constant equal to zero. We now operate on (3.11)

with the operator $\epsilon_{l m i} x_{l} \frac{\partial}{\partial x_{m}}$ to obtain the expression

$$
r \frac{\partial^{2}}{\partial r^{2}}\left\{r\left[\psi-(2+3 \alpha) a^{2} G-a^{2} \alpha r \frac{\partial G}{\partial r}\right]\right\}=\epsilon_{i j k} x_{i} \frac{\partial}{\partial x_{j}} P\left(g_{k}\right)
$$

which yields for $\psi$

$$
\psi=a^{2}\left[(2+3 \alpha) G+\alpha r \frac{\partial G}{\partial r}\right]-r^{-1} \int_{0}^{r} \rho^{-1}(r-\rho) \epsilon_{i \jmath k} x_{i} \frac{\partial}{\partial x_{j}} P\left(g_{k}\right) d \rho .
$$

Again we have set the arbitrary additive constant equal to zero in (3.15) since only derivatives of $\psi$ occur in (1.2) and (1.3). Substitution of (3.15) into (3.10) yields the result

$$
\left(r \frac{\partial}{\partial r}-c_{1}\right)\left(r \frac{\partial}{\partial r}-c_{2}\right) G=\left[4 a^{2}(\alpha+1)\right]^{-1} R
$$

where

$$
\begin{aligned}
& c_{1}=\left\{(3-\alpha)+\left[17-22 \alpha-23 \alpha^{2}\right]^{1 / 2}\right\}[4(\alpha+1)]^{-1} \\
& c_{2}=\left\{(3-\alpha)-\left[17-22 \alpha-23 \alpha^{2}\right]^{1 / 2}\right\}[4(\alpha+1)]^{-1}
\end{aligned}
$$

and

$$
R=P(f)+2\left(r \frac{\partial}{\partial r}-1\right)\left[r^{-1} \int_{0}^{r} \epsilon_{i j k} x_{i} \frac{\partial}{\partial x_{j}} P\left(g_{k}\right) d \rho\right] .
$$

For $\alpha>1 / 3$ (which corresponds to the range of $\sigma$ which is of physical interest, i.e., $-1<\sigma<1 / 2$ ) $c_{1}$ and $c_{2}$ have real part less than unity. Thus if we operate on (3.16) by $r \mathrm{\partial} / \partial r$ we obtain

$$
\left(r \frac{\partial}{\partial r}-c_{1}\right)\left(r \frac{\partial}{\partial r}-c_{2}\right) r \frac{\partial G}{\partial r}=\left[4 a^{2}(\alpha+1)\right]^{-1} r \frac{\partial R}{\partial r}
$$

an expression which can easily be integrated for $G$, giving

$$
G=\left[4 a^{2}(\alpha+1)\right]^{-1}\left\{\frac{R(0, \theta, \phi)}{c_{1} c_{2}}+\int_{0}^{r} \rho^{c_{2}-1} \int_{0}^{\rho} \rho_{1}^{c_{1}-c_{2}-1} \int_{0}^{\rho_{1}} \rho_{2}^{-c_{1}} \frac{\partial R}{\partial \rho_{2}} d \rho_{2} d \rho_{1} d \rho\right\} .
$$


If the indicated integration is performed we obtain for $c_{1} \neq c_{2}$ the expression

$$
G=\left[4 a^{2}(\alpha+1)\right]^{-1}\left\{\frac{R(r, \theta, \phi)}{c_{1} c_{2}}+\left(c_{1}-c_{2}\right)^{-1}\left[\frac{r^{c_{1}}}{c_{1}} \int_{0}^{r} \rho^{-c_{1}} \frac{\partial R}{\partial \rho} d \rho-\frac{r^{c_{2}}}{c_{2}} \int_{0}^{r} \rho^{-c_{2}} \frac{\partial R}{\partial \rho} d \rho\right]\right\} .
$$

If $c_{1}=c_{2}$ (which means that $\alpha$ satisfies the equation $17-22 \alpha-23 \alpha^{2}=0$ ) (3.20) yields upon integration

$$
G=\left[4 a^{2} c_{1}^{2}(\alpha+1)\right]^{-1}\left\{c_{1} r^{c_{1}} \int_{0}^{r} \rho^{-c_{1}} \log (r / \rho) \frac{\partial R}{\partial \rho} d \rho-r^{c_{1}} \int_{0}^{r} \rho^{-c_{1}} \frac{\partial R}{\partial \rho} d \rho+R\right\} .
$$

The function $\psi$ is now determined by insertion of (3.21) or (3.22) in (3.15), and the problem is solved.

\section{Concluding Remarks}

In a recent paper [3], the authors indicated how to obtain an explicit continuation across a spherical surface $Q$ on which $f$ and $g_{i}$ of (3.1) were zero. The decomposition (1.2), (1.3) could also be used to obtain this continuation formula. It is also possible to obtain in a straightforward way from (1.2) and (1.3) sphere theorems with various combinations of surface tractions and displacements vanishing on the surface of the sphere.

If in (1.2) and (1.3) $\alpha$ is allowed to tend to infinity while at the same time $\alpha G$ tends to a harmonic function $F$, then these decompositions may be interpreted as representation formulas for the spherical components of the velocity vector in the slow motion of viscous fluids. (See for instance [6, p. 522].)

\section{References}

[1] Bergman, S., and Schiffer, M. Kernel functions in elliptic differential equations in mathematical physics, Academic Press, New York, N Y. (1953).

[2] Bramble, J. H., Continuation of solutions of the equations of elasticity, Proc. London Math Soc. 10, 335 (1960).

[3] Bramble, J. H., and Payne, L. E., On the continuation of the solutions of the equations of elasticity by reflection, Tech. Note BN-127 University of Maryland (1960), Duke Math. J. (in press).

[4] Blokh, V. I., On the representation of the general solution of the basic equations of static elasticity for an isotropic body with the aid of harmonic functions, Prikl. Math. Mech. 22, 659 (1958).

[5] Borchardt, C. W., Gesammelte Werke, Berlin (1888).

[6] Milne-Thomson, L. M., Theoretical hydrodynamies, MacMillan Co., New York, N.Y. (1950).

[7] Sokolnikoff, I. S., Mathematical theory of elasticity, McGraw-Hill Book Co. Inc., New York, N.Y. (1956)

(Paper 65B2-54) 